\title{
Biotin-Lys-His Blocks Aggregation of RNA-binding Protein TLS, a Cause of Amyotrophic Lateral Sclerosis
}

\author{
Naomi Ueda ${ }^{1}$, Gengo Kashiwazaki ${ }^{2}$, Toshikazu Bando ${ }^{2}$, Riki Kurokawa ${ }^{1,}$ * \\ ${ }^{1}$ Division of Gene Structure and Function, Research Center for Genomic Medicine, Saitama Medical University, Saitama, Japan \\ ${ }^{2}$ Department of Chemistry, Graduate School of Science, Kyoto University, Kyoto, Japan
}

Email address:

rkurokaw@saitama-med.ac.jp (R. Kurokawa)

${ }^{*}$ Corresponding author

\section{To cite this article:}

Naomi Ueda, Gengo Kashiwazaki, Toshikazu Bando, Riki Kurokawa. Biotin-Lys-His Blocks Aggregation of RNA-binding Protein TLS, a Cause of Amyotrophic Lateral Sclerosis. Biomedical Sciences. Vol. 3, No. 4, 2017, pp. 67-77. doi: 10.11648/j.bs.20170304.11

Received: August 10, 2017; Accepted: August 30, 2017; Published: September 12, 2017

\begin{abstract}
RNA-binding protein TLS/FUS is a causative gene for amyotrophic lateral sclerosis (ALS) and frontotemporal lobar degeneration (FTLD). TLS mutations induce the propensity of TLS to form aggregates in motor neurons causing neuronal degenerative lesions to their necrosis. TLS is prone to be precipitated in high concentration around $10 \mathrm{mg} / \mathrm{ml}$, while mutated TLS is suspected to be precipitated even lower or physiological concentration in the motor neurons. An unidentified agent from infections would cause formation of the precipitation of TLS through their surface antigens. The precipitation driven with agents might be attribute to a small compound appeared on the surface. Biotinylated isoxazole (BISOX) has been reported to be precipitated with divergent RNA-binding proteins including TLS in nuclear extracts of cultured mammalian cells. We have published a molecular model for crystal formation of BISOX with TLS providing a plat form for searching novel regulators to precipitate TLS in neuronal disorders. Because BISOX is an artificial compound, we have further explored to obtain naturally occurring agents to induce the TLS precipitation and generated a conceivable biological compound, biotin-Lys-His (BLH). We have examined the precipitation of BLH with HeLa cell nuclear extract, but did not detect any TLS signal. Then, we add BLH to reaction of BISOX with TLS, and serendipitously observed a robust inhibitory effect of BLH on the formation of crystal of BISOX with TLS. We employed in silico analysis to show how BLH blocks the crystal formation of BISOX with TLS. The computational analysis of the events presented a model that BLH should be incorporated into the crystal formation of BISOX but some steric hindrance placed by BLH blocks growing of the crystal of BISOX and TLS. These results provide the potentiality that BLH should block the aggregate formation of TLS in ALS, leading to a seed for drug discovery against ALS, although it needs future endeavor to find more compounds to have effect on the TLS aggregation.
\end{abstract}

Keywords: TLS, FUS, Biotinylated Isoxazole, Biotin-Lys-His, Low Complexity Domain, Amyotrophic Lateral Sclerosis

\section{Introduction}

RNA-binding protein TLS (translocated in liposarcoma)/ FUS (fused in sarcoma) is a causative gene for amyotrophic lateral sclerosis (ALS) [1, 2] and frontotemporal lobar degeneration (FTLD) [3]. Clinical symptoms of ALS are characterized by the impairment of up and lower motor neurons, indicate muscle atrophy, and gradually spread to the muscle of whole body including respiratory system. At the late stage of the ALS, patients suffering from the difficulty of speaking and breathing terminally die from the respiratory failure $[4,5]$. More than $90 \%$ of ALS is sporadic disease in which no genetic background is identified $[4,5]$.
Around $10 \%$ of ALS is familial disease. The causative genes of familial ALS reported are the superoxide dismutase-1 [6], TDP-43 [7], and TLS [1, 2]. We have been focusing on TLS from a view point of gene expression regulation. Mutated forms of TLS genes indicate the similar pathology with other ALS causative genes, dislocation into cytoplasm from nucleus and the anomalous cytoplasmic inclusions. TLS binds RNA in specific and non-specific manner, and plays pivotal roles on RNA metabolisms in processing, and transport of RNA [8, 9]. Mutations in TLS might cause these RNA metabolic functions, suggesting its function as a mediator for biologically active RNA molecules. 
Biology of RNA has been highlighting more impact on modern science extending the previous views for the RNA metabolism that is just oriented to a step of gene expression at the process of the Central Dogma [10-19]. The extensive works on transcriptome inspections of the human genome-derived RNAs enlighten huge numbers of RNAs transcribed from noncoding regions of the human genome [20-22]. Most of the unidentified transcripts are found to be unannotated long noncoding RNAs (lncRNAs) whose biological activity remains mostly unidentified [23]. These lncRNAs are transcribed from vastly divergent sequences of the human genome, for examples, retrotransposons LINE/SINE and pseudogenes [13, 24-27]. Thus, their sequences are in full diversity and unlikely to have a common molecular mechanism behind of their biological activity. We have, however, noticed that most of RNAs should have their own RNA-binding protein partners in living cells [28]. Then, we have set RNA-binding proteins as a criterion for classification of biological activities for each lncRNA. For this end, analysis of a model system of RNA binding is employed to initiate this project. We picked RNA-binding protein TLS as a model molecule to be elucidated unannotated functions of lncRNAs [29].

Aggregation or precipitation of proteins is recently emerging as a central concerns regarding various disorders related to neurodegenerative diseases like amyotrophic lateral sclerosis (ALS) and frontotemporal lobar degeneration (FTLD) [1, 2, 8, 30-33]. It has been well recognized that precipitation of proteins in living cells causes disturbance of biological processes and gives rise to neurodegenerative diseases in the neurons. More clinical impact to these neuronal disorders enhances investigation of precipitated RNA-binding proteins that intimately link to the diseases [34-37]. We have developed experimental systems to investigate RNA-binding protein TLS to analyze the precipitation with making a model system to assess the involvement of the precipitation into the neurodegenerative diseases. Published data from others and ours indicate that BISOX effectively precipitates divergent RNA-binding proteins including TLS [38-40]. Using the BISOX precipitation system, we explored chemicals with more effect on the precipitation than BISOX and produced biotin-Lys-His (BLH), derivative of BISOX. Unexpectedly, BLH induced no significant precipitation of TLS from NE, while it inhibited the precipitation. This inspired us to obtain a drug to suppress the aggregate of TLS in neuronal disorders elicited with RNA-binding proteins including TLS. Therefore, we decided to perform in silico analysis of blocking the TLS precipitation with BISOX building up a three-dimensional model.

We undertook analysis in silico to examine interaction of BLH with TLS. Previous our data indicates that addition of BISOX into HeLa cell nuclear extract (NE) induces precipitation of TLS [40]. The low complexity (LC) domain (1-165), N-terminus of TLS is supposed to bind the surface of the BISOX crystals forming the beta-strand structure. According to the model, TLS should be incorporated into the
BISOX crystal during formation of microcrystal of BISOX.

In this manuscript, we demonstrated that BLH blocks the BISOX precipitation with TLS. This result presents the hypothesis that BLH should interfere with TLS or BISOX in the crystal formation to block the crystal formation. To reveal a mechanism, we produced a three-dimensional model to examine interaction among TLS, BLH, and BISOX and obtained the data showing that BLH is incorporated into growing crystal of BISOX with TLS and blocks further formation of the crystal. This prediction suggests that unidentified chemicals suppress the precipitation of RNA-binding protein that is involved in the neurodegenerative diseases. These results give us an insight for developing unprecedented therapeutics for ALS leading to cure.

\section{Materials and Methods}

\subsection{Materials}

Screening the chemical library to obtain chemical compounds to induce mouse embryonic stem cells into myocardial cell lineages presented a chemical compound, isoxazole [41]. Biotinylated isoxazole (BISOX), 6-(((5-(2-Thienyl)-1,2-oxazol-3-yl)carbonyl)amino)hexyl, 5-(2-oxohexahydro-1H-thieno[3,4-d]imidazol-4-yl)pentanoat e (biotin-isoxazole; BISOX) was produced following the protocols [40].

HeLa cell nuclear extract was prepared as previously described protocol [28, 29, 42].

\subsection{General Chemical Technologies}

Reagents and solvents were purchased from standard suppliers and used without further purification. Automated polyamide synthesis was performed on a PSSM-8 system (Shimadzu) by Fmoc chemistry [43, 44]. HPLC purification was performed with a JASCO PU-2080 Plus pump, a UV-2075 Plus detector (254 nm), an MX-2080-32 mixer and a DG-2080-54 degasser. A Chemcobond 5-ODS-H column (4.6 x 150 mm; Chemco Plus Scientific, Osaka, Japan) was used. The mobile phase was a gradient of acetonitrile with trifluoroacetic acid $(0.1 \%, \mathrm{v} / \mathrm{v}$ in water) at a flowrate of 1.0 $\mathrm{mL}$ min-1. ESI-TOFMS data were obtained on a BioTOF II (Bruker Daltonics).

\subsection{Synthesis of BLH (Biotin-Lys-His)}

From $55 \mathrm{mg}$ of Fmoc-His (Trt)-Wang resin $(0.32 \mathrm{meq} / \mathrm{g}$, PEPTIDES INTERNATIONAL), Boc-Lys(Fmoc)-OH (WATANABE CHEMICAL IND., LTD.) and D-biotin (nacalai tesque) were coupled stepwise. After the resin was shaken for $30 \mathrm{~min}$ at room temperature in a solution of trifluoroacetic acid (nacalai tesque) $950 \mu \mathrm{L}$, triisopropylsilane (fluorochem) $25 \mu \mathrm{L}$ and water $25 \mu \mathrm{L}$, the filtrate was dripped into Et2O to obtain the crude powder. The product was purified by HPLC to yield $5.8 \mathrm{mg}$ of white powder $(11.4 \mu \mathrm{mol}$, $71 \%$ ). ESI-TOFMS m/z calculated for C22H36N7O5S $+[\mathrm{M}+$ $\mathrm{H}]+510.2493$, found 510.2536 . 


\subsection{Protein Analysis}

SDS-polyacrylamide gel electrophoresis was performed with $10 \%$ gels following coomassie brilliant blue staining [28]. Western blotting was done with anti-TLS monoclonal antibody from the BD bioscience, 611385 with the dilution ratio 1:2000 using standard protocol shown previously [28].

\subsection{In Silico Analyses}

The structural coordinate data of the BISOX crystal (Accession number 873064) [38] was obtained from Cambridge $\quad$ Structural Database: CSD; http://www.ccdc.cam.ac.uk. A model of the main chains of the theoretical beta strand structure was constructed with the parameters setting at the dihedral angles $\Phi$ and $\Psi$ set at $120^{\circ}$ using the software Swiss-Pdb viewer [45]. The beta strand model was docked into the crystals of BISOX molecules by superimposing the beta strand model on the BISOX molecules with Waals (The Altif laboratory, Inc.). Structural models of beta strand with the side chain (Y, Q, and S) were built using Swiss-PdbViewer [45]. The side chain rotamers with no steric conflict and proper hydrogen bonds between the side chain of beta strand structure and BISOX molecules were screened, and the energy minimization was performed to optimize the conformations [46]. The divergent conformations of the BISOX and BLH molecules were constructed with the chemical structural modeling software Marvin (ChemAxon, Budapest, Hungary). We obtained the data of the BISOX crystal (Accession number 873064 ) from Cambridge Structural Database: CSD; http://www.ccdc.cam.ac.uk. CSD has been administrated with the Cambridge Crystallographic Data Center. The database contains the data regarding crystals of organic chemical compounds and metalorganic chemical compounds We obtained the crystal structural data consisting of its crystal lattice and atomic coordinate of asymmetry unit.

\section{Results}

\subsection{BLH Blocks the Precipitation of TLS with BISOX}

We successfully reproduced previous data of the precipitation of proteins from HeLa cell nuclear extract (NE) with BISOX (Figure 1A, Upper panel) [40]. Moreover, incubation of NE with BISOX at $4^{\circ} \mathrm{C}$ reproducibly induced the precipitation of RNA-binding proteins TLS, in a dose dependent manner (Figure 1A, Lower panel). Recently, we have designed and generated a novel compound named BLH which was expected to have the similar capability to precipitate TLS. BLH was incubated with NE at the same condition as the BISOX experiment, but much less amount of precipitated proteins and also TLS were observed (Figure 1B). We used higher concentration up to $400 \mu \mathrm{M}$ of $\mathrm{BLH}$, but did not detect any increment of the TLS precipitation, indicating that BLH is not another candidate for the agent to precipitate TLS. Then, we examined additive or synergistic effect of BLH on the precipitation of TLS with BISOX upon incubation both together. Unpredictably, we had neither additive nor synergistic effect of BLH on the BISOX precipitation of TLS, but observed that BLH blocks the TLS precipitation with BISOX (Figure 1C). BLH remarkably reduced the BISOX precipitation of TLS at the same concentration as BISOX, and fully removed the precipitation at the ten times more concentration than BISOX, indicating the efficient competition of BLH against BISOX on the TLS precipitation. The result presents a potency of BLH as a drug against ALS with a novel mechanism.

In order to consolidate the result, we used the purified GST-TLS for incubation with both BLH and BISOX to see if BLH blocks the precipitation. In this experiment, we have confirmed the inhibitory effect of BLH on the TLS precipitation with BISOX, indicating the effect of $\mathrm{BLH}$ is direct to TLS without any assistance by any other protein in NE (Figure 1D). These data indicate that BLH blocks the TLS precipitation with BISOX, suggesting that it should be a seed for a drug against the related diseases by suppressing the TLS precipitation in the affected neurons. Then, we decided to pursuit for a molecular mechanism for this inhibition of BLH to the TLS precipitation with BISOX using a three-dimensional model.

\section{A}

Coomassie Blue Staining

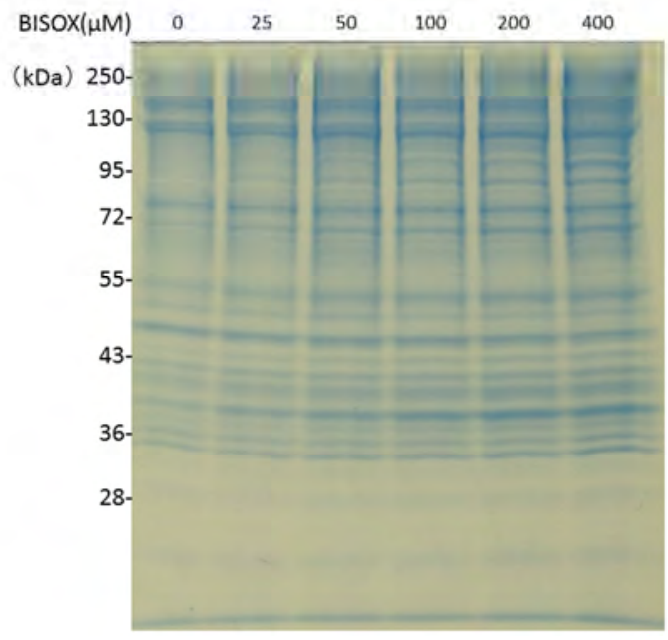

Western Blot

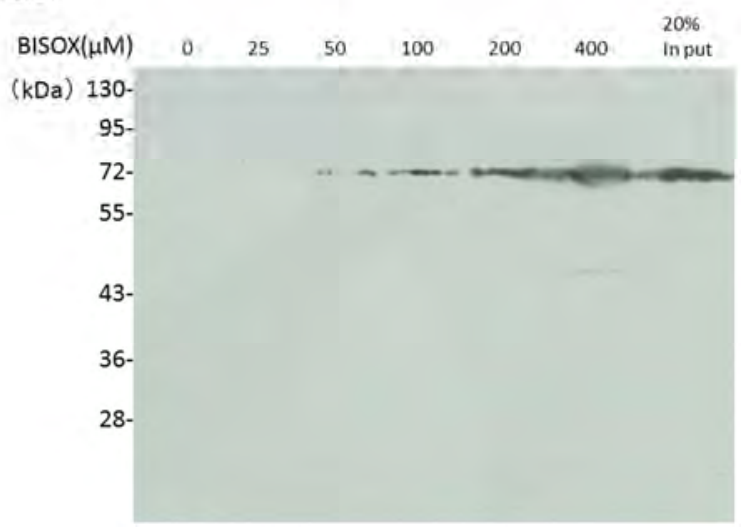




\section{B}

Coomassie Blue Staining

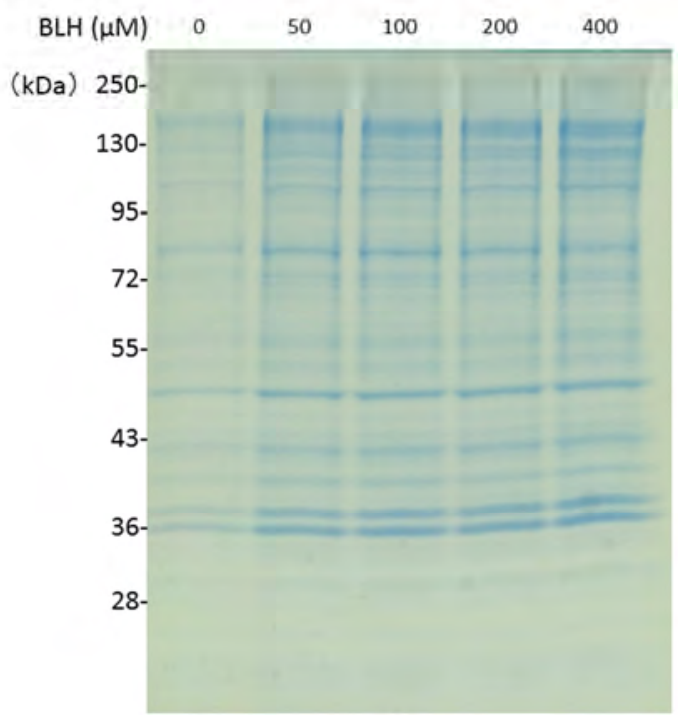

Western Blot

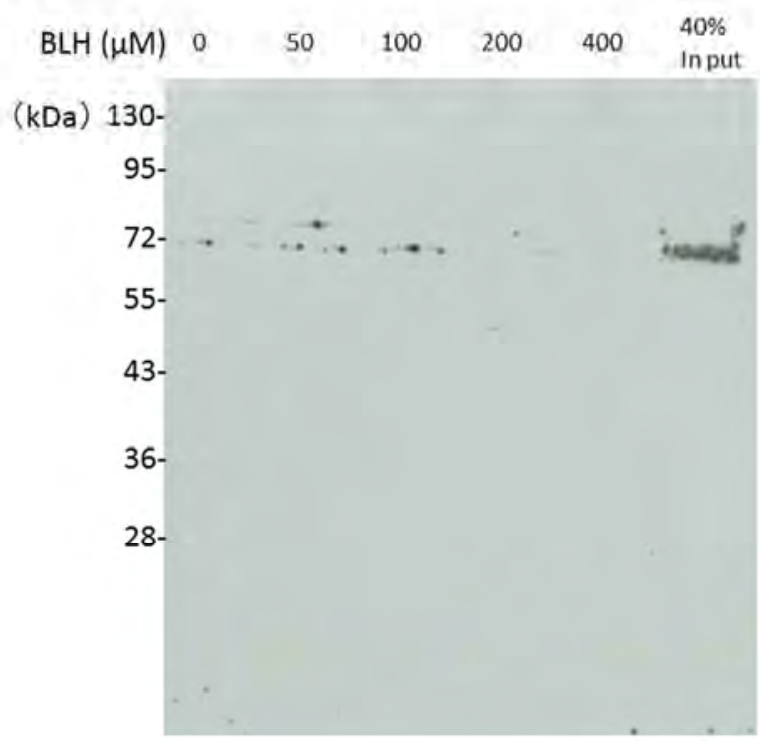

\section{C}

Western Blot

\begin{tabular}{|c|c|c|c|c|c|c|c|c|}
\hline $\mathrm{DMSO}(\mu \ell)$ & - & 1 & - & - & - & - & - & \\
\hline $\operatorname{BISOX}(\mu \mathrm{M})$ & $\cdot$ & $\cdot$ & 200 & $\cdot$ & 200 & 200 & 200 & $\begin{array}{l}20 \% \\
\text { In put }\end{array}$ \\
\hline BLH $(\mu \mathrm{M})$ & - & - & - & 200 & 200 & 1000 & 2000 & \\
\hline
\end{tabular}

(kDa) $130-$

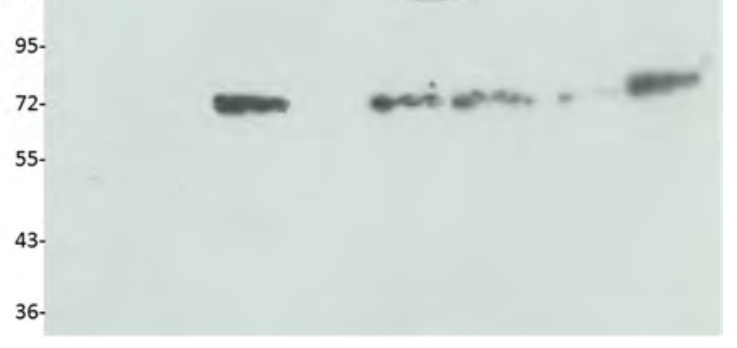

$\mathrm{D}$

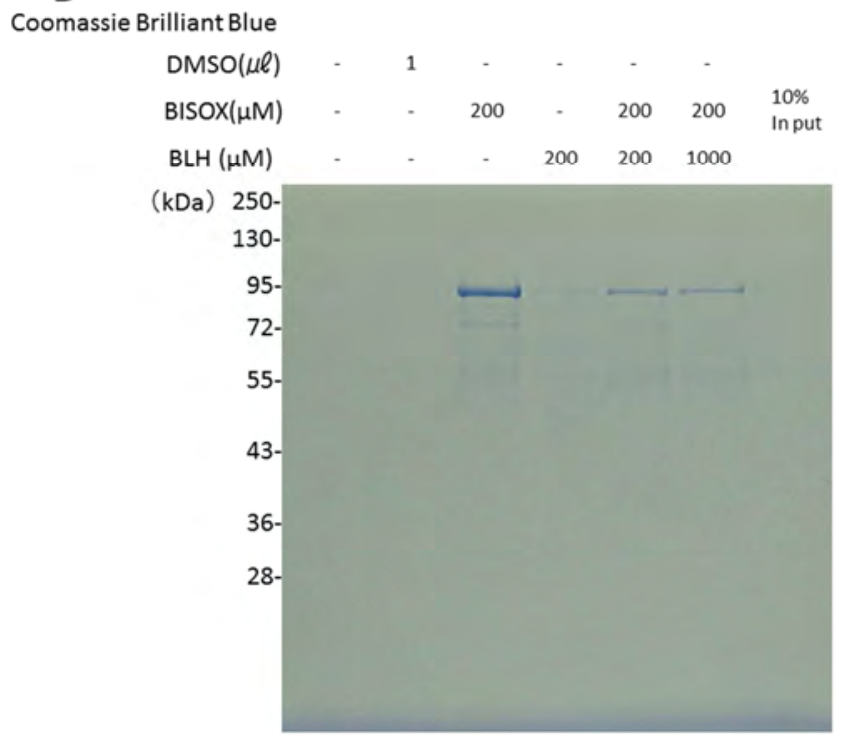

Figure 1. BLH blocks the precipitation of the BISOX with TLS

(A) BISOX effectively precipitates TLS from nuclear extract of HeLa cells in a dose-dependent manner. Coomassie blue staining (Upper panel). Western blot with TLS antibody (Lower Panel).

(B) BLH does not have any significant precipitation of TLS. Coomassie blue staining (Upper panel). Western blot with TLS antibody (Lower Panel).

(C) BLH abrogates the TLS precipitation with BISOX from the nuclear extract

(D) BISOX also makes precipitation of purified GST-TLS

\subsection{Three-Dimensional Model of BLH}

We generated the three-dimensional model of BLH in order to investigate its interaction with TLS and BISOX. Assuming that BLH is as a monomer because of no experimental evidence for its crystal formation in solution, we generated the model. The structural formula and the model are shown (Figure 2A). BLH forms a structure comprising of binding of histidine to biocytin containing biotin and lysine. The data regarding the steric conformation of biocytin, were obtained from the chemical database ZINC (http://zinc.docking.org: $\quad$ ZINC4096827). The three-dimensional model of BLH was generated by MarvinSketch (ChemAxon Ltd.) based upon the three-dimensional structural data of biocytin. Generating conformers, stable conformations of BLH are selected (top 10: Figure 2B). The top ten conformations selected are shown (Figure 2B). These are predicted as naturally stable conformations as a monomer. The biotin moiety forms variety of conformations in contrast to the Lys-His moiety.

In the BISOX crystal structure, two BISOX molecules positions at an antiparallel orientation, forming the hydrogen bonds between the carbonyl group of the biotin moiety and the amid group of the isoxazole moiety (showing with red arrows: Figure 2B) [40]. These carbonyl and amid groups should form a beta strand-like hydrogen bond on their binding to the LC domain of TLS. In the structural formula and steric conformation, BLH turned out to have the carbonyl and amid groups at the similar potion as BISOX. The 
distances between the oxygen atom of the carbonyl group and the nitrogen atom of the amid group, are $10.00 \AA$ for BISOX, and $9.82 \AA$ for BLH, respectively, fitting well the distance in the model of the theoretical beta strand $(9.33 \AA)$ of the polyglycine.

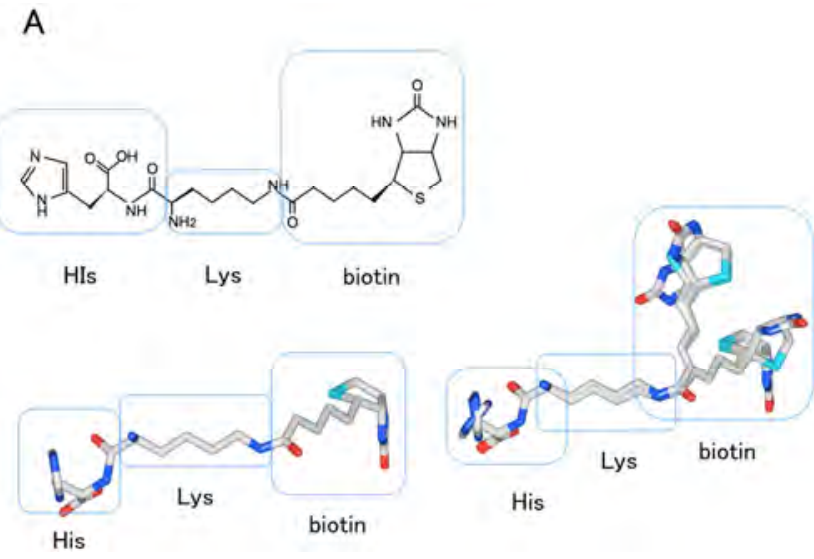

B
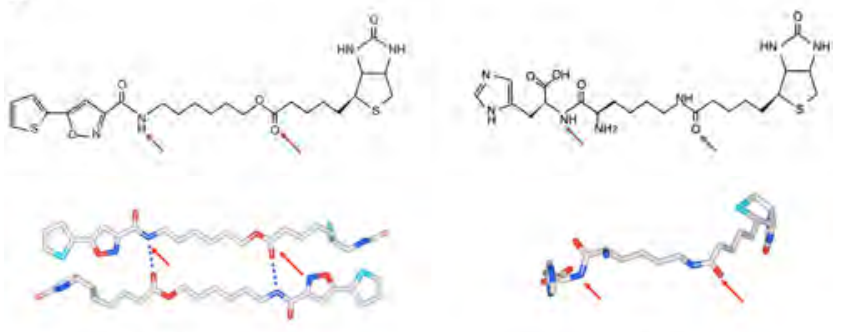

BISOX

BLH

Figure 2. Three-dimensional structure of $B L H$.

(A) The structural formula and three-dimensional model of BLH (Left panel); Superimposing of different conformations of BLH (Right panel).

(B) Comparison of BISOX and BLH structures

\subsection{The Model of Binding of BLH to the Low Complexity (LC) Regions of TLS}

Previous our data indicate that the main and side chains of the LC domain of TLS have potential to bind the BISOX crystal [40]. In our published model, the main chain of the LC domain of TLS forms the hydrogen bonds with BISOX, as similar to the beta strand. The residues of tyrosine (Y), glutamine (Q), and serine (S), which are abundant in the LC domain of TLS, are suitable for binding to the moiety of biotin in BISOX.

First of all, we explored possibility of a direct binding of BLH to TLS. Modelling the structures, we tested if the main chain of the LC domain of TLS should bind BLH as forming the beta strand and if the side chain of the beta strand of TLS should bind the biotin moiety of BLH.

\subsubsection{Interaction of the Main Chain with the Beta Strand of TLS}

BLH possesses the carbonyl and amid groups contributing to the beta strand-like bindings at the similar position as BISOX (Figure 2A and Figure 3A). Based on the three-dimensional structure of the beta sheet (PDB ID: $43 \mathrm{WBU}$ ) of TLS, we have generated a model of a complex of BLH with the beta strand of TLS, by placing BLH to form the hydrogen bonds with the main chains of the beta strand, mimicking the BISOX complex with TLS (Figure 3A). The model shows that the carbonyl and amid groups of BLH feasibly have a position to form hydrogen bonds with the main chains of the beta sheet. Although the biotin moiety of BLH is capable of forming divergent conformation, it has no steric hindrance with the main chain of the beta-strand. These data suggest that BLH should form the hydrogen bonds with the beta-strand of TLS in naturally occurring conformations.

\subsubsection{Interaction of BLH with the Side Chain of the Beta Strand of TLS}

Next, we examine if BLH interacts with the side chain of the TLS beta-strand. Previous our data suggest that the biotin moiety of BISOX should not only bind the main chain of TLS, but also the side chains of Y, Q, and S, that densely localize over the LC domains of TLS. Similarly, binding of the biotin moiety of BLH was examined to bind TLS using the model of the BLH complex with the TLS beta-strand.

We produced the three-dimensional models of BLH with several different conformations and its complex with the beta-strand of TLS (Figure 3B). In the models, the biotin moiety of BLH should form several conformations. In the BLH bound to the main chain of the beta-strand, the oxygen and the nitrogen atoms of the biotin moiety locate far from the side chains of $\mathrm{Y}, \mathrm{Q}$, and $\mathrm{S}$. In any conformation, the distance from the oxygen atom or the nitrogen atom to the side chains of the beta-strand is too distant to form hydrogen bond (the closest: 8.8A). These data indicate that the biotin moiety is improbable to bind the side chains of the beta-strand in naturally occurring conformation, although BLH could form hydrogen bond to the main chain of the beta-strand.

To assess steric hindrance to binding of the biotin moiety to the side chain of the beta-strand, we made a three-dimensional model showing hydrogen bond of the biotin of BLH with the side chains of the beta-strand by rotation of the rotamer of the biotin moiety. Upon rotation, energy increases in the model to accommodate the hydrogen bonds of the side chain of the beta-strand with the biotin generated by rotating the rotamer, compared to the original model. For example, we obtained a model forcing the biotin moiety towards the tyrosine residue of the beta-strand to form hydrogen bond by rotating the rotamer (Figure 3C). In this conformation, energy of BLH increased up to $19.4 \mathrm{kcal} / \mathrm{mol}$ compared to original one, revealing that BLH forms a forced conformation. The hydrogen bonds of BLH with the main chains is unlikely to compensate the increase loaded by the forced conformation with the hydrogen bond of the biotin to the side chains of the beta-strand because of the binding energy of hydrogen bond is only $4 \mathrm{kcal} / \mathrm{mol}$. BLH binds the main chain of the beta-strand, but is not able to bind the side chains at the same time, because in the case of its binding to both chains BLH needs to form the forced 
conformation shown above.

A

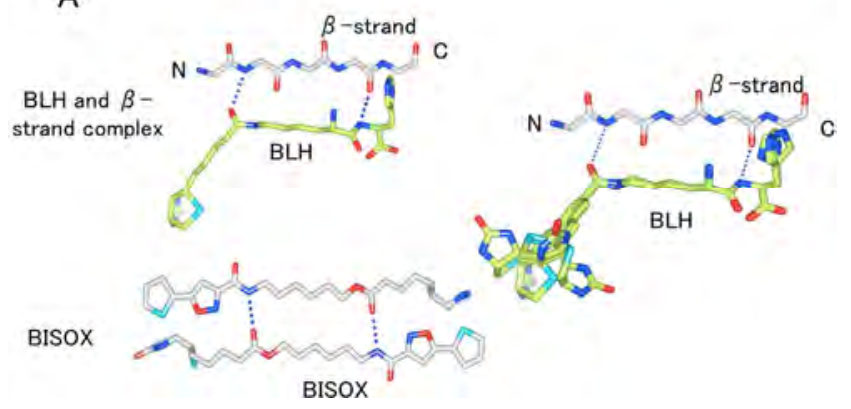

B

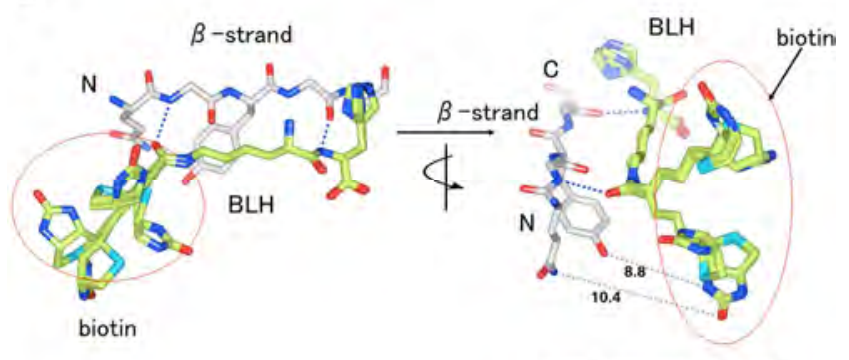

Interatom distance $[\AA]$

C

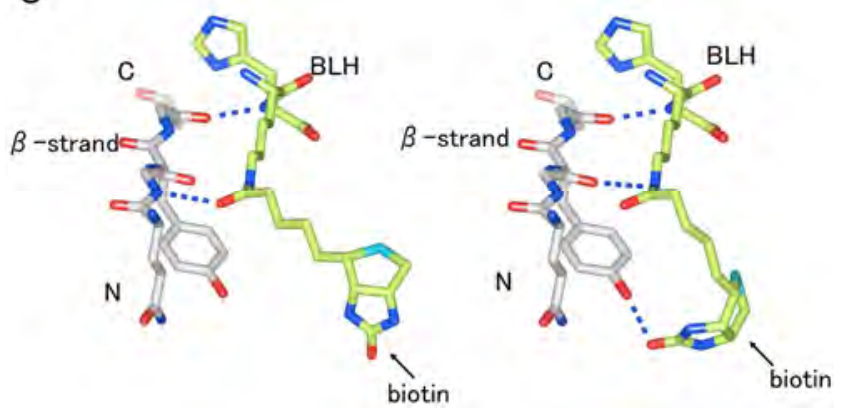

Figure 3. Various interactions of BLH.

(A) Bindings of BLH (green) to the main chains of the TLS

The carbonyl and amid groups of BLH locates to form hydrogen bonds with the main chains of the beta-strand (Left panel)

Superimposing of different structures of BLH, without any steric hindrance (Right panel)

(B) Binding of BLH (green) with the side chains of the beta-strand

(C) Binding of BLH on rotation of its biotin moiety to the side chains of the beta-strand

There is conformational disorder in rotation of the rotamer of biotin moiety of BLH to make binding of the tyrosine residue of the beta-strand of TLS to the biotin of BLH

\subsection{Interaction of $B L H$ with BISOX}

We employed our published three-dimensional model of the crystal of BISOX with superimposing the BLH structure to uncover any possible interaction of BLH with BISOX [40].

\subsubsection{Model of the Complex of BLH with BISOX}

We produced the model of the complex of BLH with BISOX by adapting the three-dimensional model of BLH based on the backbone of BISOX, to our previous multilayer model of the BISOX crystal [40]. The model shows that a BLH molecule is well incorporated into the surface with a convex-concave shape, a valley and mountain shape, without any steric hindrance (Figure 4A). It is likely that the biotin moiety of BLH binds well as comparable to that of BISOX. The lysine portion of BLH should bind as similar to the carbon chains of BISOX. The histidine portion of BLH locates as floating because it does not have enough space to fit well the groove of the BISOX crystal (Figure 4A).

\subsubsection{The Interaction of BLH with BISOX}

Our present models demonstrate that a BLH molecule should bind the surface of the grooves of the BISOX crystal form the beta-strand like hydrogen bonds with consecutive a BISOX molecule in the layers of the crystal (Figure 4B). It was also shown that the biotin moieties of BISOX and BLH form hydrogen bonds between the layers of the crystal, indicating that BLH should bind the surfaces of the BISOX crystals in a manner similar to BISOX.

In the interactions between layers, it is indicated that several hydrophobic interactions of the carbon chains of lysine and biotin portions to that of BISOX (Figure 4C). The hydrophobic interaction between BLH and BISOX should contribute to enhancement of affinity in both BLH and BISOX, although it is less forceful than that in the crystals of BISOX alone. These predictions suggest that the affinity of $\mathrm{BLH}$ to BISOX is higher than that to TLS because of no hydrophobic interaction of the LC domain of TLS with BLH.

The histidine portion of BLH protrudes upwards from the surface of the BISOX crystal owing to no space to be placed in the groove. This is a major difference between the BLH incorporation into the BISOX crystal and the BISOX alone crystal growing. This protrusion of the histidine portion abolishes the surface of the crystal, and blocks the further incorporation of BLH into the growing crystal.

The model of the complex of BLH and BISOX indicates the hydrogen bonds of the beta-strand like structures and the biotin moiety of these two molecules, and the hydrophobic interaction between layers, suggesting that BLH binds to BISOX with higher affinity than TLS, suggesting that BLH could compete with TLS on formation of the crystal.

A

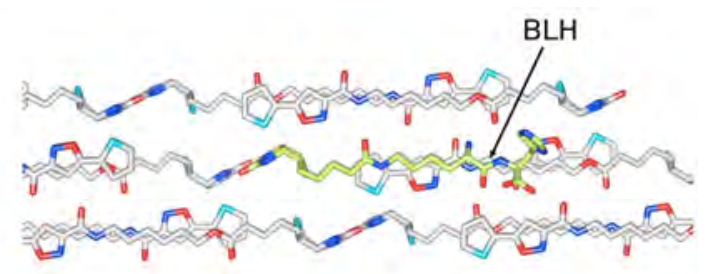

Side

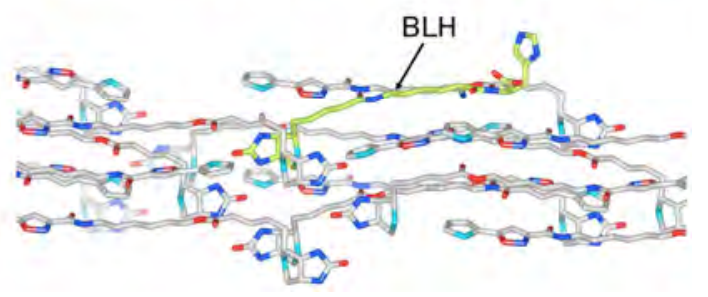


B

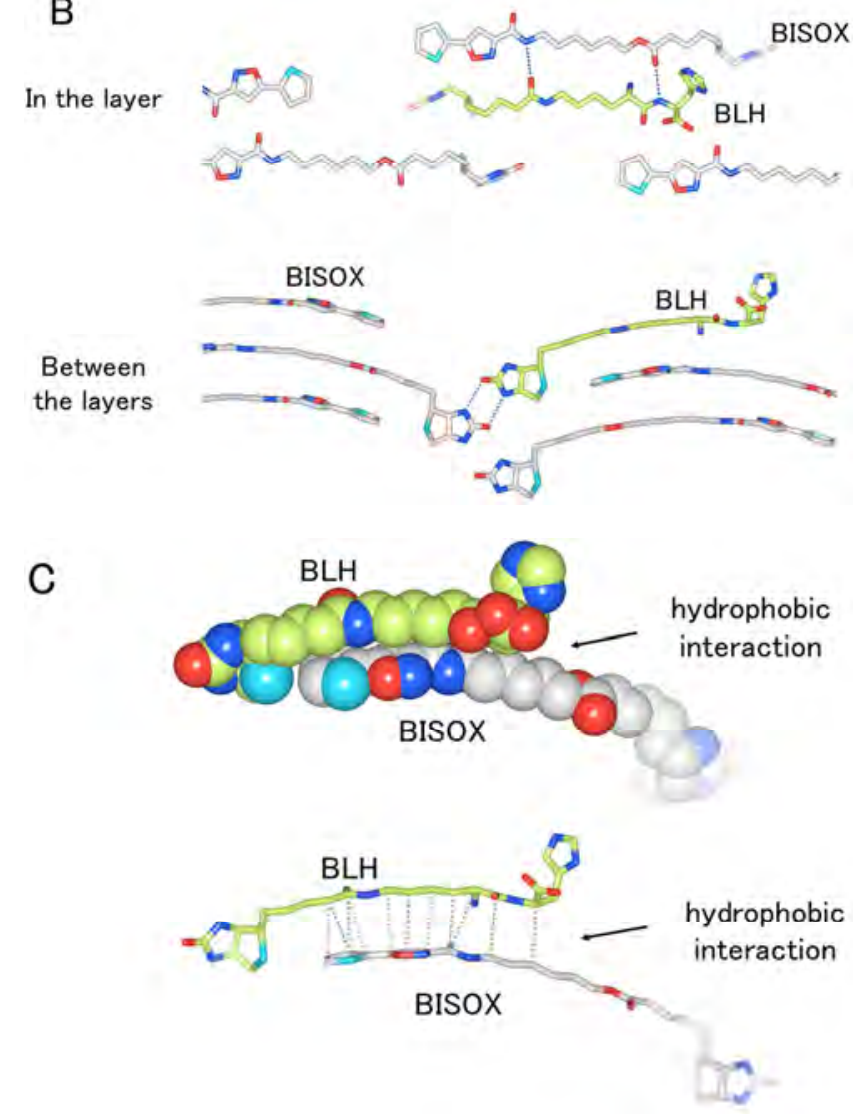

Figure 4. The interaction of BLH with the BISOX crystal.

(A) The three-dimensional model of the BLH (green) complex with BISOX

(B) The hydrogen bonds between layers in the crystal formation of BISOX with BLH

BLH forms the hydrogen bonds between the biotins with BISOX similar to BISOX alone

In the layer (Upper panel); between the layers (Lower panel)

(C) The hydrophobic interactions between layers in the co-crystal formation of BLH (green) with BISOX

\section{Discussion}

Precipitation of RNA-binding proteins is one of major causes for the neurodegenerative diseases. Forming polymers of TLS through its LC domain, the resulting polymer of TLS is precipitated out of the solution and forms aggregates $[9,47$, 48]. There is a growing list of RNA-binding proteins including TDP-43, hnRNP1, and TLS with mutations, that are involved in ALS and FTLD of sporadic and inherited forms $[32,49,50]$. At this manuscript, we picked TLS as a model molecule to analyze the pathogenic pathways of ALS. Neuropathological events caused by the TLS mutants are the deposition of insoluble precipitations of the mutant RNA-binding proteins in the nucleus and cytoplasm of neurons in the brain and spinal cord of ALS patients.

It has been focused on the LC domains of divergent proteins including numerous RNA-binding proteins [51,52].
The LC domain consists of repeats of several amino acids and only forms a specific conformation upon its interactions with specific partners like proteins and nucleic acid. In the pathological process, the LC domains of the $\mathrm{N}$ terminus of TLS drives its phase transitions, reversibly shifting between dispersed, liquid droplet, and hydrogel-like phases [31, 38, 53]. The mutation of TLS severely limits this ability to repeatedly shift between these phases. Actually, the mutated TLSs induce the propensity of TLS to condense into poorly soluble, stable, fibrillary hydrogel-like assemblies. These stably irreversible TLS aggregates, which selectively capture other RNA-binding proteins, impair local RNA-binding protein granule functions, and attenuate novel protein syntheses in the axon terminals of cultured neurons [31]. Modulation of these protein phase transitions might be a possible therapeutic target.

However, our focus is on the molecular dissection of the precipitation of TLS, because this is certainly early step of the series of events of the disease, in which its onset could be terminated. The dynamic phase transitions of TLS have been one of hot topics in studies of ALS. Manipulation of these phases transition might be a tractable therapeutic target. Practically, chemical reaction is more manipulable step to be executed via a drug. Therefore, we have been investigating BISOX as a TLS-precipitating reagent and obtained the blocking agent BLH against the TLS precipitation. Analyzing the blocking process of the TLS precipitation with BLH, we would dissect the molecular mechanism of the TLS precipitation and obtained protocol to repress the precipitation leading to development of unprecedented therapeutics against ALS. To learn more about the molecular mechanism of blocking the TLS precipitation of BISOX by $\mathrm{BLH}$, we constructed conformational models related to these molecules.

The model of the complex of BLH with the beta-strand indicates that BLH barely binds the side chains of the beta-strand, although BLH could form two hydrogen bonds with the main chains of the beta-strand. It is unlikely that five resides of the fluctuating LC domain without forming any specific conformation captures BLH just with only two hydrogen bonds to it. The model of the complex of BISOX with BLH indicates that BLH binds BISOX through the hydrogen bonds with beta-strands and biotin moieties, also with hydrophobic interactions between layers. These results show that BLH should bind the BISOX crystal more than the TLS LC domain. It is also likely that BLH binds BISOX with higher affinity than TLS because BLH has the distinctive hydrophobic interaction with BISOX that is not observed with TLS. Our previous model indicated that TLS is incorporated into the surface of the BISOX crystals [40]. Therefore, BLH reduces incorporation of TLS into the BISOX crystal, suggesting that BLH should block the precipitation of TLS in addition of BLH into the BISOX crystal forming with TLS (Figure 5A). 
A

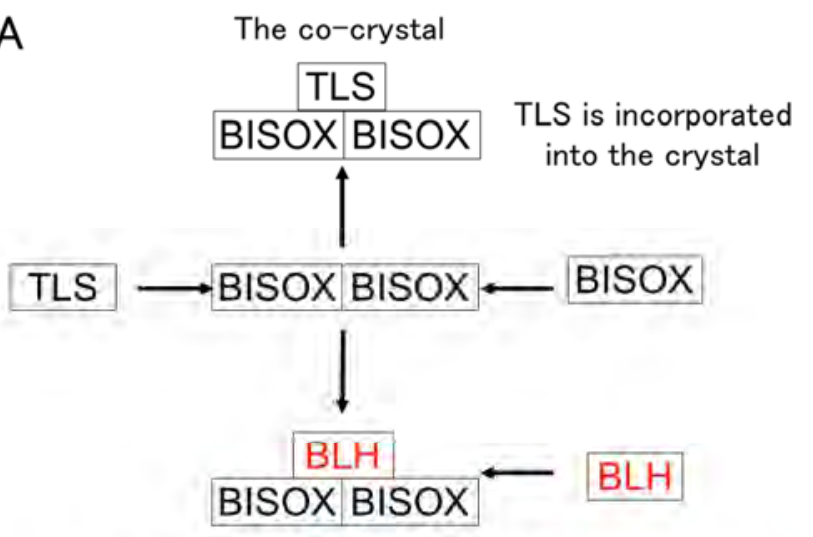

BLH bound the BISOX crystal

BLH blocks growing of the co-crystal of BISOX with TLS
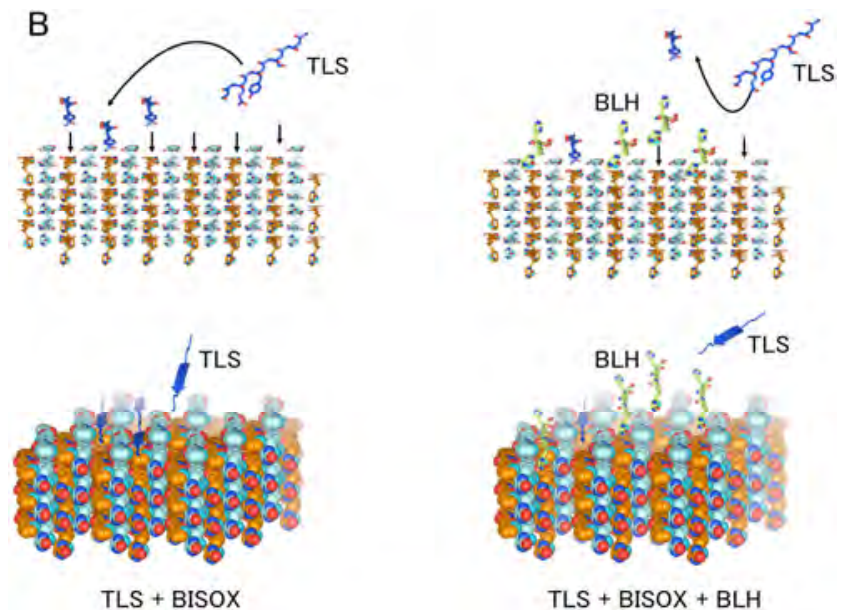

Figure 5. Repression of the TLS and BISOX crystal formation with BLH.

(A) Mechanism of the BLH repression of the BISOX crystal formation with TLS

(B) Three-dimensional model of the BLH repression of the BISOX crystal formation with TLS

BISOX forms microcrystals in aqueous solution, while upon addition of TLS to the solution it is incorporated into the surface of the crystal and forms the co-crystal (Figure 5AB). There are two possibilities how BLH represses the co-crystallization of BISOX with TLS. (1) BLH captures TLS and block formation of the co-crystal with BISOX. (2) BLH competes with TLS to bind the crystal of BISOX and binds the crystal to force TLS out of the crystal (Figure 5B). Our model in this manuscript indicates that BLH should bind the BISOX crystal more firmly than TLS, confirming the possibility (2). BLH forms the beta strand-like hydrogen bonds and also hydrogen bonds with the biotin moiety of BISOX, comparable to TLS. Furthermore, BLH forms hydrophobic interaction with BISOX that TLS does not, suggesting that BLH has higher affinity for the BISOX crystal than TLS and that BLH binds the surface of the BISOX crystal faster and stronger than TLS (Figure 5B). BLH deprives TLS of its binding space in the BISOX crystal. It is more strenuous for TLS to occupy the space in the BISOX crystal because BLH more effectively spreads out over the surface of the crystal to block the TLS binding.

Taken together, these results show that BLH should form the crystal with BISOX with repressing the TLS incorporation into the crystal (Figure 5AB). It is possible to obtain a blocking chemical like BLH to impede the precipitation by a chemical like BISOX by addition of the blocking chemical with prompt binding to the precipitation.

Recently, it has been reported that the propensity of specific RNA sequences to form various base-pairing could induce their gelation without assistance of protein [37]. It has shown that RNP granules, for example, stress granules, P-granules, and nucleoli, are phase-separated liquid-like compartments $[54,55]$. Numerous studies have shown proteins for their capability to phase-separate and mediate the assemblies of these RNP granules [54]. Recent data have shown that sequence-specific base-pairing properties of RNAs could lead to their phase separation and gelation, and predict that this kind of phenomena could contribute to physiological granule assemblies in living cells [37]. It has also been shown that intermolecular base-pairing should result in the aggregation and sequestration of RNA into nuclear foci in the neurodegenerative diseases [37]. Remarkably, addition of molecules to the experimental systems to disrupt the formation of complementary interactions between RNA bases, resulted in abruption of the formation of RNA foci, showing that these base-paring is essential for the RNA gelation. These data raise the potency that unprecedented therapeutics for disrupting RNA-RNA base pairing could be developed for better patient cares. This strategy is analogous to our present data that BLH blocks the TLS precipitation with BISOX leading to a novel drug. Therefore, agents to block macromolecular aggregations of proteins and also RNA are a potential seed for related neuronal diseases and could lead to powerful therapeutics to complete care for the diseases.

\section{Conclusion}

In this manuscript, we demonstrated that BLH blocks the BISOX precipitation with TLS. The data present a hypothesis that BLH interacts with TLS or BISOX in the crystal formation to repress the crystal formation. To explore a mechanism, we produced a three-dimensional model to test interaction among TLS, BLH, and BISOX. The model indicated that BLH is incorporated into the growing crystal of BISOX with TLS and blocks further formation of the crystal. This prediction suggests that unidentified chemicals suppress the precipitation of RNA-binding protein that is involved in the neurodegenerative diseases. These results give us an insight for developing unprecedented therapeutics for ALS leading to cure.

\section{Acknowledgements}

The authors would thank for excellent technical supports by Mr. Sei-ichi Aikawa and Fumiko Matsuzawa (Altif Laboratories Inc.). This study was supported by Grant-in-Aid for Scientific Research (B: nos22390057; 25293073). This 
work was also supported in part by a grant-in-aid for "Support Project of Strategic Research Center in Private Universities" from the Ministry of Education, Culture, Sports, Science and Technology (MEXT) to Saitama Medical University Research Center for Genomic Medicine.

\section{References}

[1] Vance C, Rogelj B, Hortobagyi T, De Vos KJ, Nishimura AL, Sreedharan J, Hu X, Smith B, Ruddy D, Wright P, et al. (2009) Mutations in FUS, an RNA processing protein, cause familial amyotrophic lateral sclerosis type 6. Science 323, 1208-1211, doi: 323/5918/1208 [pii]10.1126/science. 1165942

[2] Kwiatkowski TJ, Jr., Bosco DA, Leclerc AL, Tamrazian E, Vanderburg CR, Russ C, Davis A, Gilchrist J, Kasarskis EJ, Munsat T, et al. (2009) Mutations in the FUS/TLS gene on chromosome 16 cause familial amyotrophic lateral sclerosis. Science 323, 1205-1208, doi: 323/5918/1205 [pii]10.1126 /science.1166066.

[3] Mackenzie IR \& Neumann M (2016) Molecular neuropathology of frontotemporal dementia: insights into disease mechanisms from postmortem studies. Journal of neurochemistry 138 Suppl 1, 54-70, doi: 10.1111/jnc.13588.

[4] Ludolph AC, Brettschneider J \& Weishaupt JH (2012) Amyotrophic lateral sclerosis. Current opinion in neurology 25, 530-535, doi: 10.1097/WCO.0b013e328356d328.

[5] Arbab M, Baars S \& Geijsen N Modeling motor neuron disease: the matter of time. Trends Neurosci 37, 642-652, doi: 10.1016/j.tins.2014.07.008.

[6] Rosen DR, Siddique T, Patterson D, Figlewicz DA, Sapp P, Hentati A, Donaldson D, Goto J, O'Regan JP, Deng HX, et al. (1993) Mutations in $\mathrm{Cu} / \mathrm{Zn}$ superoxide dismutase gene are associated with familial amyotrophic lateral sclerosis. Nature 362, 59-62, doi: 10.1038/362059a0.

[7] Sreedharan J, Blair IP, Tripathi VB, Hu X, Vance C, Rogelj B, Ackerley S, Durnall JC, Williams KL, Buratti E, et al. (2008) TDP-43 mutations in familial and sporadic amyotrophic lateral sclerosis. Science 319, 1668-1672, doi: $10.1126 /$ science. 1154584 .

[8] Lagier-Tourenne C \& Cleveland DW (2009) Rethinking ALS: the FUS about TDP-43. Cell 136, 1001-1004, doi: S0092-8674(09)00263-3 [pii]10.1016/j.cell.2009.03.006.

[9] Taylor JP, Brown Jr RH \& Cleveland DW (2016) Decoding ALS: from genes to mechanism. Nature 539, 197-206, doi: 10.1038/nature20413.

[10] Kurokawa R (2015) Long Noncoding RNAs. In, pp. 257. Springer.

[11] Lipovich L, Tarca AL, Cai J, Jia H, Chugani HT, Sterner KN, Grossman LI, Uddin M, Hof PR, Sherwood CC, et al. (2014) Developmental changes in the transcriptome of human cerebral cortex tissue: long noncoding RNA transcripts. Cereb Cortex 24, 1451-1459, doi: 10.1093/cercor/bhs414.

[12] Derrien T, Johnson R, Bussotti G, Tanzer A, Djebali S, Tilgner H, Guernec G, Martin D, Merkel A, Knowles DG, et al. (2012) The GENCODE v7 catalog of human long noncoding RNAs: analysis of their gene structure, evolution, and expression. Genome research 22, 1775-1789, doi: 10.1101/gr.132159.111.
[13] Carninci P \& Kasukawa T \& Katayama S \& Gough J \& Frith MC \& Maeda N \& Oyama R \& Ravasi T \& Lenhard B \& Wells C, et al. (2005) The transcriptional landscape of the mammalian genome. Science 309, 1559-1563, doi: 309/5740/1559 [pii]10.1126/science.1112014.

[14] Khalil AM, Guttman M, Huarte M, Garber M, Raj A, Rivea Morales D, Thomas K, Presser A, Bernstein BE, van Oudenaarden A, et al. (2009) Many human large intergenic noncoding RNAs associate with chromatin-modifying complexes and affect gene expression. Proc Natl Acad Sci U S A 106, 11667-11672, doi: 0904715106 [pii]10.1073 /pnas.0904715106.

[15] Necsulea A, Soumillon M, Warnefors M, Liechti A, Daish T, Zeller U, Baker JC, Grutzner F \& Kaessmann H (2014) The evolution of IncRNA repertoires and expression patterns in tetrapods. Nature 505, 635-640, doi: 10.1038/nature12943.

[16] Chi KR (2016) Finding function in mystery transcripts. Nature 529, 423-425, doi: 10.1038/529423a.

[17] Kurokawa R (2012) Generation of Functional Long Noncoding RNA Through Transcription and Natural Selection. In Regulatory RNAs, pp. 151-174. Springer.

[18] Djebali S, Davis CA, Merkel A, Dobin A, Lassmann T, Mortazavi A, Tanzer A, Lagarde J, Lin W, Schlesinger F, et al. (2012) Landscape of transcription in human cells. Nature 489, 101-108, doi: 10.1038/nature11233.

[19] Hon C-C, Ramilowski JA, Harshbarger J, Bertin N, Rackham OJL, Gough J, Denisenko E, Schmeier S, Poulsen TM, Severin J, et al. (2017) An atlas of human long non-coding RNAs with accurate 5' ends. Nature 543, 199-204, doi: 10.1038 /nature21374 http://www.nature.com/nature/journal/v543/n7644/abs/nature2 1374.html\#supplementary-information.

[20] Kurokawa R (2011) Long noncoding RNA as a regulator for transcription. Prog Mol Subcell Biol 51, 29-41, doi: 10.1007/978-3-642-16502-3_2.

[21] Kurokawa R (2011) Promoter-associated long noncoding RNAs repress transcription through a RNA binding protein TLS. Advances in experimental medicine and biology 722, 196-208, doi: 10.1007/978-1-4614-0332-6_12.

[22] Kurokawa R (2015) Initiation of Transcription Generates Divergence of Long Noncoding RNAs. In Long Noncoding RNAs, pp. 69-91. Springer.

[23] Kurokawa R, Rosenfeld MG \& Glass CK (2009) Transcriptional regulation through noncoding RNAs and epigenetic modifications. RNA Biol 6, 233-236, doi: 8329 [pii].

[24] Carninci P, Sandelin A, Lenhard B, Katayama S, Shimokawa K Ponjavic J, Semple CA, Taylor MS, Engstrom PG, Frith MC, et al. (2006) Genome-wide analysis of mammalian promoter architecture and evolution. Nat Genet 38, 626-635, doi: ng1789 [pii]10.1038/ng1789.

[25] Duret L, Chureau C, Samain S, Weissenbach J \& Avner P (2006) The Xist RNA gene evolved in eutherians by pseudogenization of a protein-coding gene. Science 312, 1653-1655, doi: 312/5780/1653 [pii]10.1126/science.1126316.

[26] Johnsson P, Ackley A, Vidarsdottir L, Lui W-O, Corcoran M, Grandér D \& Morris KV (2013) A pseudogene long noncoding RNA network regulates PTEN transcription and translation in human cells. Nature structural \& molecular biology 20, 440-446, doi: $10.1038 / \mathrm{nsmb} .2516$. 
[27] Scarola M, Comisso E, Pascolo R, Chiaradia R, Maria Marion R, Schneider C, Blasco MA, Schoeftner S \& Benetti R (2015) Epigenetic silencing of Oct4 by a complex containing SUV39H1 and Oct4 pseudogene IncRNA. Nat Commun 6, 7631, doi: 10.1038/ncomms8631.

[28] Yoneda R, Suzuki S, Mashima T, Kondo K, Nagata T, Katahira $M$ \& Kurokawa R (2016) The binding specificity of Translocated in LipoSarcoma/FUsed in Sarcoma with lncRNA transcribed from the promoter region of cyclin D1. Cell \& bioscience 6, 4, doi: 10.1186/s13578-016-0068-8.

[29] Wang X, Arai S, Song X, Reichart D, Du K, Pascual G, Tempst P, Rosenfeld MG, Glass CK \& Kurokawa R (2008) Induced ncRNAs allosterically modify RNA-binding proteins in cis to inhibit transcription. Nature 454, 126-130, doi: nature06992 [pii]10.1038/nature06992.

[30] Turner MR, Hardiman O, Benatar M, Brooks BR, Chio A, de Carvalho M, Ince PG, Lin C, Miller RG, Mitsumoto H, et al. (2013) Controversies and priorities in amyotrophic lateral sclerosis. The Lancet Neurology 12, 310-322, doi: 10.1016/S1474-4422(13)70036-X.

[31] Murakami T, Qamar S, Lin JQ, Schierle GS, Rees E, Miyashita A, Costa AR, Dodd RB, Chan FT, Michel CH, et al. (2015) ALS/FTD Mutation-Induced Phase Transition of FUS Liquid Droplets and Reversible Hydrogels into Irreversible Hydrogels Impairs RNP Granule Function. Neuron 88, 678-690, doi: 10.1016/j.neuron.2015.10.030.

[32] Lagier-Tourenne C, Polymenidou M \& Cleveland DW (2010) TDP-43 and FUS/TLS: emerging roles in RNA processing and neurodegeneration. Hum Mol Genet 19, R46-64, doi: ddq137 [pii]10.1093/hmg/ddq137.

[33] Sun S, Ling S-C, Qiu J, Albuquerque CP, Zhou Y, Tokunaga S, Li H, Qiu H, Bui A, Yeo GW, et al. (2015) ALS-causative mutations in FUS/TLS confer gain and loss of function by altered association with SMN and U1-snRNP. Nat Commun 6, doi: $10.1038 /$ ncomms 7171 .

[34] DeJesus-Hernandez M, Mackenzie IR, Boeve BF, Boxer AL, Baker M, Rutherford NJ, Nicholson AM, Finch NA, Flynn H, Adamson J, et al. (2011) Expanded GGGGCC hexanucleotide repeat in noncoding region of C9ORF72 causes chromosome 9p-linked FTD and ALS. Neuron 72, 245-256, doi: 10.1016/j.neuron.2011.09.011.

[35] Renton AE, Majounie E, Waite A, Simon-Sanchez J, Rollinson S, Gibbs JR, Schymick JC, Laaksovirta H, van Swieten JC, Myllykangas L, et al. (2011) A hexanucleotide repeat expansion in C9ORF72 is the cause of chromosome 9p21-linked ALS-FTD. Neuron 72, 257-268, doi: 10.1016/j.neuron.2011.09.010.

[36] Jucker M \& Walker LC (2013) Self-propagation of pathogenic protein aggregates in neurodegenerative diseases. Nature 501, 45-51, doi: 10.1038/nature12481.

[37] Jain A \& Vale RD (2017) RNA phase transitions in repeat expansion disorders. Nature 546, 243-247, doi: 10.1038 /nature22386.

[38] Kato M, Han TW, Xie S, Shi K, Du X, Wu LC, Mirzaei H, Goldsmith EJ, Longgood J, Pei J, et al. (2012) Cell-free formation of RNA granules: low complexity sequence domains form dynamic fibers within hydrogels. Cell 149, 753-767, doi: 10.1016/j.cell.2012.04.017.

[39] Han TW, Kato M, Xie S, Wu LC, Mirzaei H, Pei J, Chen M,
Xie Y, Allen J, Xiao G, et al. (2012) Cell-free formation of RNA granules: bound RNAs identify features and components of cellular assemblies. Cell 149, 768-779, doi: 10.1016/j.cell.2012.04.016.

[40] Kurokawa R \& Bando T (2016) Three-Dimensional Structure of RNA-Binding Protein TLS Co-Crystallized with Biotinylated Isoxazole. Biomedical Sciences 2, 1-10, doi: 10.11648/j.rnat.20160201.11.

[41] Sadek H, Hannack B, Choe E, Wang J, Latif S, Garry MG, Garry DJ, Longgood J, Frantz DE, Olson EN, et al. (2008) Cardiogenic small molecules that enhance myocardial repair by stem cells. Proceedings of the National Academy of Sciences 105, 6063-6068, doi: 10.1073/pnas.0711507105.

[42] Song X, Wang X, Arai S \& Kurokawa R (2012) Promoter-associated noncoding RNA from the CCND1 promoter. Methods in molecular biology 809, 609-622, doi: 10.1007/978-1-61779-376-9_39.

[43] Asamitsu S, Kawamoto Y, Hashiya F, Hashiya K, Yamamoto M, Kizaki S, Bando T \& Sugiyama H (2014) Sequence-specific DNA alkylation and transcriptional inhibition by long-chain hairpin pyrrole-imidazole polyamide-chlorambucil conjugates targeting CAG/CTG trinucleotide repeats. Bioorg Med Chem 22, 4646-4657, doi: 10.1016/j.bmc.2014.07.019.

[44] Kawamoto Y, Sasaki A, Hashiya K, Ide S, Bando T, Maeshima K \& Sugiyama H (2015) Tandem trimer pyrrole-imidazole polyamide probes targeting 18 base pairs in human telomere sequences. Chemical Science 6, 2307-2312, doi: $10.1039 / \mathrm{c} 4 \mathrm{sc} 03755 \mathrm{c}$.

[45] Guex N \& Peitsch MC (1997) SWISS-MODEL and the Swiss-PdbViewer: an environment for comparative protein modeling. Electrophoresis 18, 2714-2723, doi: 10.1002/elps.1150181505.

[46] Rahman MM, Kitao S, Tsuji D, Suzuki K, Sakamoto J-I, Matsuoka K, Matsuzawa F, Aikawa S-I \& Itoh K (2013) Inhibitory effects and specificity of synthetic sialyldendrimers toward recombinant human cytosolic sialidase 2 (NEU2). Glycobiology 23, 495-504, doi: 10.1093/glycob/cws221.

[47] Berchowitz Luke E, Kabachinski G, Walker Margaret R, Carlile Thomas M, Gilbert Wendy V, Schwartz Thomas U \& Amon A (2015) Regulated Formation of an Amyloid-like Translational Repressor Governs Gametogenesis. Cell 163, 406-418, doi: http://dx.doi.org/10.1016/j.cell.2015.08.060.

[48] Schwartz Jacob C, Wang X, Podell Elaine R \& Cech Thomas R (2013) RNA Seeds Higher-Order Assembly of FUS Protein. Cell Reports 5, 918-925, doi: http://dx.doi.org/10.1016/j.celrep.2013.11.017.Kurokawa R (2015) Initiation of Transcription Generates Divergence of Long Noncoding RNAs. In Long Noncoding RNAs, pp. 69-91. Springer.

[49] Kim HJ, Kim NC, Wang YD, Scarborough EA, Moore J, Diaz Z, MacLea KS, Freibaum B, Li S, Molliex A, et al. (2013) Mutations in prion-like domains in hnRNPA2B1 and hnRNPA1 cause multisystem proteinopathy and ALS. Nature 495, 467-473, doi: 10.1038/nature11922.

[50] Ling SC, Albuquerque CP, Han JS, Lagier-Tourenne C, Tokunaga S, Zhou H \& Cleveland DW (2010) ALS-associated mutations in TDP-43 increase its stability and promote TDP-43 complexes with FUS/TLS. Proc Natl Acad Sci U S A 107, 13318-13323, doi: 1008227107 [pii]10.1073 /pnas.1008227107. 
[51] Alberti S, Halfmann R, King O, Kapila A \& Lindquist S (2009) A systematic survey identifies prions and illuminates sequence features of prionogenic proteins. Cell 137, 146-158, doi: 10.1016/j.cell.2009.02.044.

[52] King OD, Gitler AD \& Shorter J (2012) The tip of the iceberg: RNA-binding proteins with prion-like domains in neurodegenerative disease. Brain Research 1462, 61-80, doi: 10.1016/j.brainres.2012.01.016.

[53] Elbaum-Garfinkle S \& Brangwynne CP (2015) Liquids, Fibers, and Gels: The Many Phases of Neurodegeneration. Dev Cell 35, 531-532, doi: 10.1016/j.devcel.2015.11.014.
[54] Hyman AA, Weber CA \& Julicher F (2014) Liquid-liquid phase separation in biology. Annu Rev Cell Dev Biol 30, 39-58, doi: 10.1146/annurev-cellbio-100913-013325.

[55] Wojciechowska M \& Krzyzosiak WJ (2011) Cellular toxicity of expanded RNA repeats: focus on RNA foci. Human molecular genetics 20, 3811-3821, doi: 10.1093/hmg/ddr299. 